\title{
Urinary Podocalyxin and Lupus Nephritis Disease Activity
}

\author{
Ashraf Hussein Mohamed ${ }^{1}$, Mahmoud Awad ${ }^{1}$, Asmaa Enein ${ }^{1}$, \\ Mona G. Balata ${ }^{2}$, Alaa Mohamed Alsalawy ${ }^{3}$, Emad Elmasry ${ }^{4}$ \\ Departments of Internal Medicine, Mansoura University ${ }^{1}$; Rheumatology ${ }^{2}$, Ain Shams University; \\ Physical Medicine \& Rheumatology ${ }^{3}$, Tanta University; Clinical Pathology ${ }^{4}$, Mansoura University; Egypt
}

\begin{abstract}
Background: Lupus nephritis (LN) is a common and serious complication in systemic lupus erythematosus (SLE) and is associated with significant mortality and morbidity of SLE patients. The conventional laboratory markers used in clinical practice such as serum complement levels and double-stranded DNA antibodies are unreliable indicators of LN as they lack both sensitivity and specificity for prediction of active or relapsing LN. Studies have shown that podocyte injury occurs in the early stages of glomerular damage in LN, and that quantification of podocyturia could be used as a marker for active disease. Podocalyxin (PCX) is probably the most frequently used marker protein for podocyturia. Aim of the work: To investigate urinary podocalyxin (u-PCX) as a marker of lupus nephritis (LN) disease activity. Methods: 63 patients with clinical and biopsy proven LN were recruited and divided into two groups; 35(55.56\%) patients with active LN \& $28(44.44 \%)$ patients with inactive LN. Estimation of u-PCX/creatinine ratio, urine protein /creatinine ratio (PCR), erythrocyte sedimentation rate, $\mathrm{C}$ reactive protein, serum albumin, serum complement $\mathrm{C} 3$ and $\mathrm{C} 4$, anti-double stranded DNA antibody titers, serum creatinine, estimated glomerular filtration rate and renal biopsy were done. Results: Patients with active LN had significant higher levels of PCR (1159.38 \pm 724.37 Vs 332.29 \pm 145.10$)$, u-PCX (109.18 \pm 28.21 Vs 67.93 \pm 8.24$)$, and SLEDAI-2K renal score $(6.83 \pm 2.16$ Vs $2.0 \pm 1.17)(\mathrm{P}<0.001)$. However, active $\mathrm{LN}$ group had significant lower serum albumin $(2.87 \pm 0.51 \mathrm{Vs}$ $3.61 \pm 0.22, \mathrm{P}<0.001)$. In active $\mathrm{LN}$; $\mathrm{u}-\mathrm{PCX}$ correlated positively with PCR $(\mathrm{r}=0.516, \mathrm{p}=0.002)$, SLEDAI-2k (renal) $(\mathrm{r}=0.568$, $\mathrm{p}<0.001)$, ads DNA ab $(\mathrm{r}=0.362, \mathrm{p}<0.032)$ and negatively with serum albumin $(\mathrm{r}=-0.421, \mathrm{p}=0.014)$.Moreover, SLEDAI-2k renal score was negatively correlated with serum albumin $(\mathrm{r}=-0.710, \mathrm{P}<0.001)$. Conclusion: $\mathrm{u}-\mathrm{PCX}$ could be served as a marker of LN disease activity. [Egypt J Rheumatology \& Clinical Immunology, 2016; 4(1): 23-31]
\end{abstract}

Key Words: Disease activity- Lupus nephritis - Podocytes - Urinary podocalyxin.

\section{INTRODUCTION}

Lupus nephritis (LN) is a common and serious complication in systemic lupus erythematosus (SLE) and is associated with significant mortality and morbidity of SLE patients ${ }^{1}$. Clinically, proteinuria and haematuria are characteristic features in patients with $\mathrm{LN}$ and have traditionally been thought to be the result of immune complex deposition and endocapillary proliferation causing a disruption to the filtration barrier. However, in a subset of proteinuric lupus patients, there is no evidence of the typical immune complexes and instead there appears to be extensive podocyte effacement ${ }^{2}$.

The conventional laboratory markers used in clinical practice such as serum complement levels and double-stranded DNA antibodies are unreliable indicators of $\mathrm{LN}$ as they lack both sensitivity and specificity for prediction of active or relapsing LN. Moreover, serum creatinine is also unsatisfactory as a marker as significant renal damage can occur before it rises $^{3}$. Other laboratory tests such as proteinuria and urinary sediments are also non-specific markers ${ }^{4}$.

Podocytes are highly differentiated cells that line the outside of the glomerular capillary and are formed of a body with extending major processes that further branch into foot processes separated by a slit diaphragm. They play an important role in the generation of urine by separating proteins from the aqueous part of the blood ${ }^{5,6}$.

Studies have shown that podocyte injury occurs in the early stages of glomerular damage in $\mathrm{LN}^{7}$, and that quantification of podocyturia could be used as a marker for active disease ${ }^{8,9}$ Podocalyxin (PCX) is probably the most frequently used marker protein for podocyturia. It is a $140-\mathrm{kDa}$-sized polyanionic sialoprotein, localized in plasma membrane of podocytes ${ }^{10}$. We therefore investigated the clinical significance of urinary podocalyxin (u-PCX) as a marker of $\mathrm{LN}$ disease activity in SLE patients.

\section{SUBJECTS AND METHODS}

This cross-sectional study was carried out on 63 SLE patients recruited from the Nephrology and Rheumatology clinics in Riyadh national hospital in Riyadh, KSA; All patients (59 females \& 4 males) with clinical and biopsy proven LN were included ${ }^{11}$. This study was carried out between January 2012 and August 2014. 
Exclusion criteria: We excluded those patients with end stage renal disease or had undergone renal transplantation, pregnant patients, patient known to have chronic kidney disease due to other causes rather than LN, patients refuse renal biopsy and those refuse to give consent.

\section{The Included patients:}

Patients were divided into two groups based on the presence or absence of LN activity; $35(55.56 \%)$ patients with active LN \& $28(44.44 \%)$ patients with inactive LN. Patients with relapse/ flare of LN were included in the active group.

\section{All patients were subjected to:}

Through clinical, medication history.

Laboratory parameters which included: body mass index $\left(\mathrm{BMI} \mathrm{kg} / \mathrm{m}^{2}\right)$, urine analysis by dipstick, urine microscopy, urine protein/creatinine ratio (PCR), erythrocyte sedimentation rate (ESR), $\mathrm{C}$ reactive protein (CRP) serum albumin, serum complement C3 and $\mathrm{C} 4$, anti-double stranded DNA antibody (ads DNA $a b$ ), serum creatinine and estimated glomerular filtration rate (eGFR) using the Modification of Diet in Renal Disease (MDRD) formula. Renal ultrasound was done for all patients at the start of the study to exclude possible renal causes of renal impairment. Fresh urine samples obtained for u-podocalyxin (uPCX) testing were immediately centrifuged to remove sediments and then frozen in aliquots at $-80^{\circ} \mathrm{C}$ for $\mathrm{u}$ PCX testing.

A written consent from patients in the study and an approval from the local ethics and scientific committees were obtained.

\section{SLE disease activity index assessment}

SLE Disease activity was assessed using the SLE Disease Activity Index (SLEDAI) ${ }^{12}$. The renal score was based on the presence of any of the following abnormal parameters in the urinalysis in the absence of stones, urinary tract infection or other causes: haematuria ( $\geq 5$ red blood cells/ high power field (HPF), leukocyturia ( $\geq 5$ leukocytes/ HPF) and urinary casts (granular or red blood cell casts) ${ }^{12}$.

Renal disease activity was measured by SLEDAI-2k (renal) Score. It was calculated as follows; proteinuria $0.5-1 \mathrm{~g} /$ day (3 points), proteinuria 1-3 g/day ( 5 points), proteinuria $>3 \mathrm{~g} /$ day (11 points), urine RBC's > 5/hpf (3 points), and urine WBC's > $5 / \mathrm{hpf}(1 \text { point })^{13}$. LN activity (active, relapse/flare, remission and/or inactive) was assessed. ${ }^{13,14}$

\section{Renal biopsy}

It was obtained from all patients with $\mathrm{LN}$ at the start of the study for pathological classification of $\mathrm{LN}$ according to the criteria defined by the $\mathrm{WHO}^{15}$. The renal histopathological examination included light microscopic examination and immunofluorescence. The activity index (AI) and chronicity index (CI) of each biopsy specimen were also determined according to the previously accepted indices ${ }^{16}$. Evaluation of the biopsy specimens was performed by a single expert pathologist.

\section{Detection of urinary podocalyxin (u-PCX)}

Human Podocalyxin (PCX) ELISA Kit (Wuhan USCN Business Co, Hubei, P. R. China) was used for the quantitative determination of human (u-PCX) concentrations in urine. This assay employs the quantitative sandwich enzyme immunoassay technique.

Urine collection: Use a sterile container to collect urine samples (early morning samples). Remove any particulates by centrifugation for 15 minutes at $1000 \mathrm{xg}, 2-8^{\circ} \mathrm{C}$ and aliquot and store samples at $-20^{\circ} \mathrm{C}$ or $-80^{\circ} \mathrm{C}$. Centrifuge again before assaying to remove any additional precipitates that may appear after storage.

Antibody specific for podocalyxin (Purified Human PCX antibody) has been pre-coated into a microplate. Standards and samples are pipetted into the wells and any podocalyxin present is bound by the immobilized antibody. After removing any unbound substances, a biotin-conjugated antibody specific for podocalyxin is added to the wells. After washing, avidin conjugated Horseradish Peroxidase (HRP) is added to the wells.

Following a wash to remove any unbound avidin-enzyme reagent, a substrate solution is added to the wells and color develops in proportion to the amount of podocalyxin bound in the initial step. The color development is stopped and the intensity of the color is measured at wave length: $450 \mathrm{~nm}$. Detection range; this assay provided a linear plot covering a range of $3.12-200 \mathrm{ng} / \mathrm{ml}$.

Sensitivity; is typically less than $0.09 \mathrm{ng} / \mathrm{ml}$. Specificity, this assay has high specificity for detection of human podocalyxin. No significant crossreactivity or interference between human podocalyxin and analogues was observed. U-PCX levels were expressed as concentrations normalized for urine creatinine and presented as nanogram per mill mol creatinine (ng/mmol).

\section{Western blot analysis for Urinary podocalyxin}

The presence of u-PCX in urine samples (obtained after centrifugation) was confirmed by western blot analysis. The proteins in the sample were separated on 5-20\% (wt/vol.) SDS-PAGE, and then transferred (under reducing conditions) into a PVDF membrane. The membrane was incubated for $1 \mathrm{~h}$ at room temperature with monoclonal antibodies against $\mathrm{u}-\mathrm{PCX}$. The membrane was incubated with anti-mouse IgG labelled with HRP (Wuhan, Hubei Province, P. R. 
China) and finally visualized using diaminobenzidine. Specificity this assay has high specificity with less than $1 \%$ crosses reactivity with recombinant mouse Podocalyxin and recombinant human Endoglycan.

\section{Statistical Analysis}

It was performed using SPSS version 21.0 (SPSS Inc., Chicago, IL). Data are expressed as mean value \pm SD. Differences between two groups in clinical \& laboratory data were compared by t-test for normally distributed values. Correlations between u-PCX and all parameters of LN activity were assessed using Spearman's correlation coefficients. Receiver operating characteristic (ROC) curves were generated and the area under the curve (AUC) was used to measure the discriminatory capacity of u-PCX levels for identification of LN activity. The AUC for u-PCX was compared with those of the standard markers of $\mathrm{LN}$ activity. The best cut-off value for u-PCX was calculated on the basis of maximization of the Youden index (sensitivity + specificity -1$)^{17}$. Multivariate analysis was performed by means of binary logistic regression to evaluate the independent predictors of LN activity. Differences between groups were considered to be statistically significant when $\mathrm{P}<0.05$.

\section{RESULTS}

This study was conducted on 63 patients (59 females \& 4 males) with clinical and biopsy proven LN. Their mean age was (range 18-56 y) and their mean disease duration was (range 2-18 y).

Patients with active LN had significant higher levels of u-PCX (Figure 1), proteinuria, hematuria, leucocyturia, ads DNA abs, SLEDAI-2k (global) score \& SLEDAI-2k (renal) score and a significant lower serum albumin and $\mathrm{C} 4$ levels $(\mathrm{P}<0.001)$. There were no differences between the two groups as regards demographic and other clinical and laboratory data (Table 1)

However, as regard medications; there were a significant differences in the use of immunosuppressive medications between the two groups; Azathioprine $(\mathrm{P}=0.024)$ Cyclosporine or Tacrolimus $(\mathrm{P}=0.012)$ and Cyclophosphamide was used only in active LN. There were no associations between u-PCX levels and all medication use apart from with usage of intravenous Cyclophosphamide; u-PCX levels were higher in patients treated with Cyclophosphamide compared with those who were not (Table 2).

U-PCX levels and correlation with clinical and laboratory parameters

In active LN patients; U-PCX correlated positively with PCR ( $\mathrm{r}=0.515, \mathrm{P}=0.002)$, SLEDAI-2K (global) score $(\mathrm{r}=0.466, \mathrm{p}<0.001)$ and SLEDAI-2k (renal) score $(\mathrm{r}=0.585, \mathrm{p}<0.001) \quad$ (Figure 2) and negatively with serum albumin $(\mathrm{r}=-0.421, \mathrm{p}=0.014)$. More data are presented in Table (3). SLEDAI-2k (renal) was negatively correlated with serum albumin $(\mathrm{r}=-0.710, \mathrm{P}<0.001)$ and positive correlated with $\mathrm{PCR}$, hematuria \& ads-DNA abs $(\mathrm{r}=0.803, \quad \mathrm{P}<0.001$, $\mathrm{r}=0.371, \quad \mathrm{P}=0.028, \quad \mathrm{r}=0.369, \quad \mathrm{P}=0.029)$, while in inactive $\mathrm{LN}$ patients there were no correlations between u-PCX and others parameters.

\section{In western blot analysis}

U-PCX was found as granular structures in the urine precipitates following ultracentrifugation at $435,000 \mathrm{~g}$ of urine samples from patients with $\mathrm{LN}$. The presence of PCX was confirmed by western blot analysis. The precipitate obtained after centrifugation contained a typical PCX band at $165-170 \mathrm{kDa}$. No band was observed with the control antibody (monoclonal antibody against norovirus $[\mathrm{NV}]$ ) and PBS.

\section{Diagnostic performance of $\mathrm{u}-\mathrm{PCX}$ in $\mathrm{LN}$}

A nonparametric ROC curve was performed to assess the potential diagnostic values u-PCX compared to the standard markers for active $\mathrm{LN}$; the AUC for u-PCX was of $0.926(95 \%$ CI: of 0.856 0.996: $\mathrm{P}<0.001$; Figure 3). In comparison, The AUC for proteinuria was $0.940(95 \%$ CI: $0.886-0.972$ : $\mathrm{P}<0.001)$, The AUC for hematuria was $0.732(95 \%$ CI: 0.602-0.862: $\mathrm{P}=0.002$ ) and for leucocyturia was 0.664(95\% CI: 0.527-0.801: $\mathrm{P}=0.026$ ) (Figure 3A).

The AUC for SLEDAI-2K renal score was 1.00(95\% CI: 1.00-1.00: $\mathrm{p}<0.0001$ ) and the AUC for adsDNA was 0.696 (95\% CI: 0.560-0.832: $\mathrm{P}<0.008$ ), for $\mathrm{C} 4$ was 0.344 (95\% CI: 0.201-0.488: $\mathrm{P}<0.035$ ), for serum albumin 0.112 (95\% CI: 0.031-0.192:P<0.001) and for eGFR was 0.442 (95\% CI: $0.299-0.586$ : $\mathrm{p}=0.435)$ (Figure 3B).

Thus, u-PCX was better than hematuria, leucocyturia, adsDNA ab, C4, serum albumin and eGFR but was not as good as proteinuria and SLEDAI-2K renal score in predicting and diagnosing early LN activity. At a cut off value of $86.35 \mathrm{ng} / \mathrm{mmol}$ with a maximum Youden index of 0.60 , the sensitivity of U-PCX for early diagnosis of active $\mathrm{LN}$ was 0.71 with specificity of 0.89 .

\section{Independent predictor of $\mathrm{LN}$ activity}

Multiple logistic binary regressions were performed to assess the independent predictors for LN activity. UPCX and all laboratory variables relevant to LN activity with a $\mathrm{p}$ value $\leq 0.05$ (Table 3 ) were entered in the regression model. U-PCR and Proteinuria were the independent predictors of $\mathrm{LN}$ activity (Table 4). 
Mohamed, et al.: Urinary podocalyxin and lupus nephritis disease activity

Table 1. The demographic, clinical and laboratory parameters of the studied groups.

\begin{tabular}{|c|c|c|c|}
\hline \multirow{2}{*}{ Parameters } & \multicolumn{2}{|c|}{ Patients groups } & \multirow{2}{*}{ P-value } \\
\hline & Active LN (35 patients) & Inactive LN (28patients) & \\
\hline Gender (female/male) & $33 / 2$ & $26 / 2$ & NS \\
\hline Age (year) & $30.04 \pm 10.06$ & $33.57 \pm 9.27$ & NS \\
\hline $\operatorname{BMI}\left(\mathrm{kg} / \mathrm{m}^{2}\right)$ & $29.42 \pm 3.04$ & $31.18 \pm 4.73$ & NS \\
\hline Duration (year) & $6.67 \pm 4.39$ & $6.82 \pm 4.28$ & NS \\
\hline ESR (mm/hour) & $75.76 \pm 25.27$ & $25.03 \pm 11.22$ & $<0.001$ \\
\hline $\mathrm{CRP}(\mathrm{mg} / \mathrm{dl})$ & $2.62 \pm 0.93$ & $2.29 \pm 0.94$ & NS \\
\hline $\mathrm{u}-\mathrm{PCX}$ (ng/mmol ) & $109.18 \pm 28.21$ & $67.93 \pm 8.24$ & $<0.001$ \\
\hline Hematuria score & $2.57 \pm 1.06$ & $1.18 \pm 1.49$ & $<0.001$ \\
\hline Leucocyturia score & $0.69 \pm 0.47$ & $0.36 \pm 0.49$ & 0.009 \\
\hline 24 hours urinary protein & $1159.38 \pm 724.37$ & $332.29 \pm 145.10$ & $<0.001$ \\
\hline Serum albumin $(\mathrm{gm} / \mathrm{dl})$ & $2.87 \pm 0.51$ & $3.61 \pm 0.22$ & $<0.001$ \\
\hline Anti-ad DNA abs (IU/ml) & $116.08 \pm 29.35$ & $94.68 \pm 32.99$ & 0.009 \\
\hline $\mathrm{C} 3(\mathrm{mg} / \mathrm{dl})$ & $49.35 \pm 14.34$ & $54.42 \pm 17.83$ & NS \\
\hline $\mathrm{C} 4(\mathrm{mg} / \mathrm{dl})$ & $7.47 \pm 3.04$ & $10.47 \pm 5.27$ & 0.006 \\
\hline SLEDAI-2K(Global) score & $15.37 \pm 5.08$ & $11.39 \pm 4.58$ & 0.001 \\
\hline (SLEDAI-2K(Renal) score & $6.83 \pm 2.16$ & $2.04 \pm 1.17$ & $<0.001$ \\
\hline Extra renal activity score & $8.71 \pm 4.30$ & $9.89 \pm 3.95$ & NS \\
\hline Serum creatinine $(\mu \mathrm{mol} / \mathrm{l})$ & $126.70 \pm 42.89$ & $124.11 \pm 43.43$ & NS \\
\hline eGFR (ml/min) & $51.71 \pm 17.09$ & $58.06 \pm 14.90$ & NS \\
\hline
\end{tabular}

$\boldsymbol{K} \boldsymbol{g}$ kilogram, $\boldsymbol{m}^{2}$ meter square, $\boldsymbol{m} \boldsymbol{m}$ millimeter, $\boldsymbol{m g}$ milligram, $\boldsymbol{d l}$ deciliter, $\boldsymbol{g} \boldsymbol{m}$ gram, $\boldsymbol{I U}$ international unite, $\boldsymbol{n g}$ nanogram, mmol milli mole, $\mu m o l$ micromole, $\boldsymbol{m i}$ mille, $\boldsymbol{m i n}$ minute

Table 2. Histopathological and medications data of the patients groups.

\begin{tabular}{lccc}
\hline \multicolumn{1}{c}{ WHO Classes of LN no. $(\%)$} & Active LN & Non active LN & --- \\
\hline I & --- & --- & --- \\
II & --- & $2(7.14 \%)$ & 0.046 \\
III & $8(22.86 \%)$ & $14(50.00 \%)$ & 0.018 \\
IV & $21(60.00 \%)$ & $7(25.00 \%)$ & NS \\
V & $6(17.14 \%)$ & $5(17.86 \%)$ & 0.001 \\
Renal biopsy Activity index (/24) (mean \pm SD) & $15.82 \pm 2.38$ & $5.33 \pm 1.12$ & NS \\
Renal biopsy Chronicity index (/12) (mean \pm SD) & $5.67 \pm 1.68$ & $6.50 \pm 1.46$ & NS \\
Medications, no. $(\%)$ & & & NS \\
- Prednisolone & $35(100 \%)$ & $28(100 \%)$ & 0.022 \\
Hydroxychloroquine & $26(74.29 \%)$ & $23(82.14 \%)$ & NS \\
- Azathioprine & $9(25.71 \%)$ & $20(71.43 \%)$ & 0.012 \\
- Mychophenolic acid & $12(34.29 \%)$ & $8(28.57 \%)$ & --- \\
- Cyclosporine/Tacrolimus & $16(45.71 \%)$ & $6(21.43 \%)$ & NS \\
- Cyclophosphamide & $9(25.71 \%)$ & $0(0 \%)$ & $25(89.29 \%)$ \\
- Renin Angiotensin System Blockers (ACEI/ARB) & $26(74.29 \%)$ & & \\
\hline
\end{tabular}

$\overline{A C E I}$ angiotensinogen converting enzyme inhibitor, $\boldsymbol{A R B}$ Angiotensin receptor blocker 
Mohamed, et al.: Urinary podocalyxin and lupus nephritis disease activity

Table 3. Correlation between u-PCX and laboratory parameters in active LN patients.

\begin{tabular}{lcc}
\hline \multicolumn{1}{c}{ Spearman's rho variables } & r & P value \\
\hline Proteinuria & 0.516 & 0.002 \\
Hematuria & 0.252 & 0.140 \\
Leucocyturia & 0.204 & 0.239 \\
Serum albumin & -0.421 & 0.014 \\
SLEDAI (global) & 0.466 & $<0.001$ \\
SLEDAI (renal) & 0.585 & $<0.001$ \\
AdsDNA & 0.362 & 0.032 \\
C4 & -0.123 & 0.483 \\
C3 & -0.140 & 0.423 \\
eGFR & -0.207 & 0.234 \\
Serum creatinine & 0.194 & 0.322 \\
Renal biopsy activity score & 0.640 & $<0.001$ \\
CRP & 0.013 & 0.942 \\
\hline
\end{tabular}

Table 4. Logistic regression models for predictors of LN activity.

\begin{tabular}{lcccccccc}
\hline \multicolumn{1}{c}{ Parameters } & B & SE & Wald & df & Sig. & EXP $(\mathbf{B})$ & \multicolumn{2}{c}{ 95\% CI of EXP $(\mathbf{B})$} \\
\hline u-PCX & & & & & & & & \\
Proteinuria & -0.061 & .028 & 4.974 & 1 & 0.026 & 0.941 & 0.891 & 0.993 \\
Serum albumin & -0.008 & .003 & 5.991 & 1 & 0.014 & 0.992 & 0.986 & 0.998 \\
AdsDNA abs & -0.015 & 0.014 & 1.227 & 1 & 0.268 & 0.985 & 0.959 & 1.001 \\
\hline
\end{tabular}

$\boldsymbol{B}$ Standard regression coefficient; $\boldsymbol{S E}$ Standard Error; $\boldsymbol{C I}$ Confidence Interval

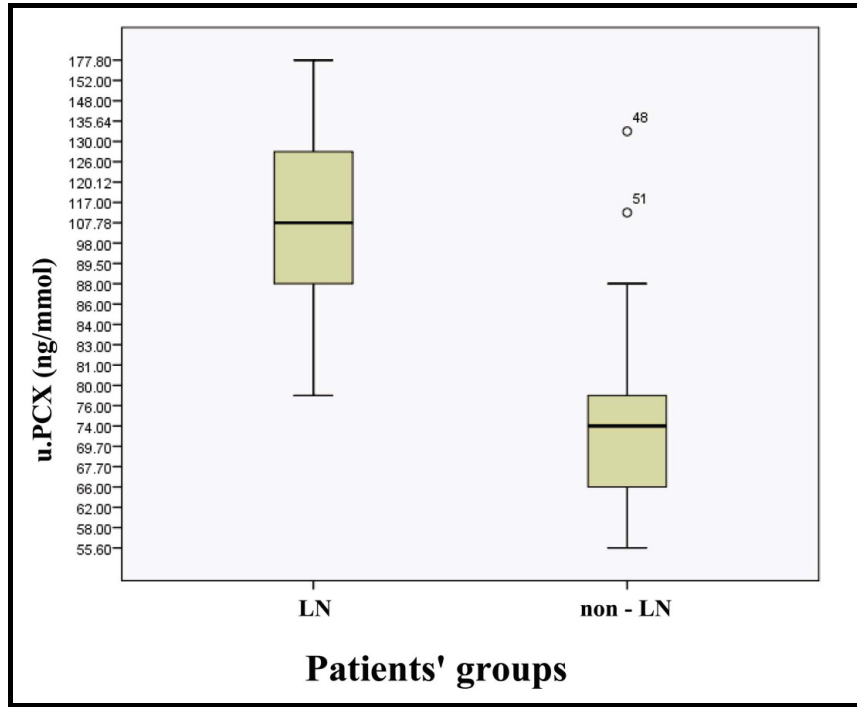

Figure 1. u-PCX levels in patients with and without active LN. 


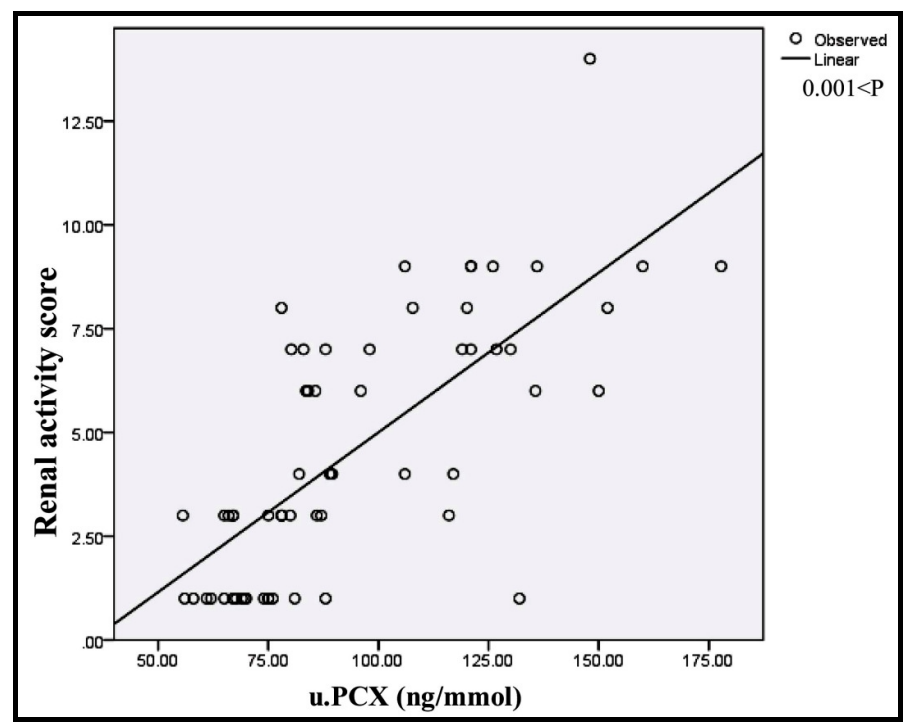

Figure 2. The correlation between u-PCX levels and SLEDAI-2K (renal)

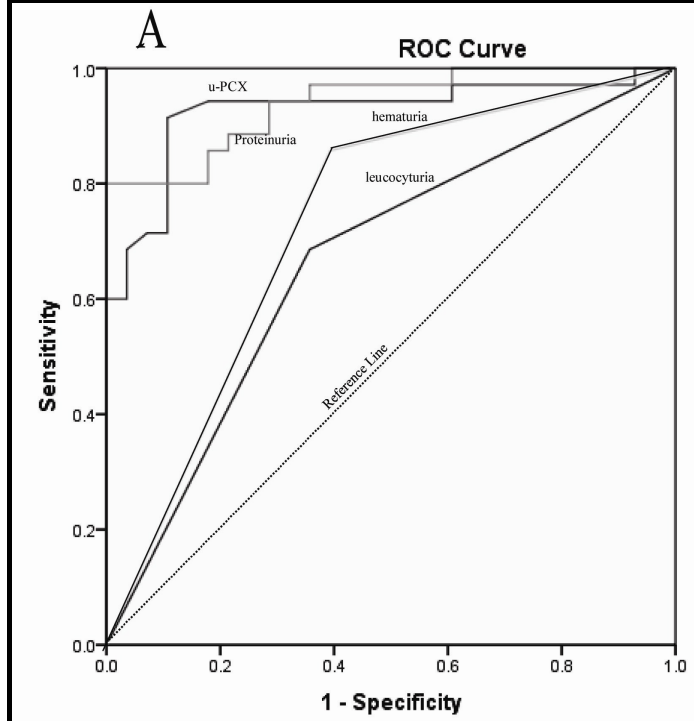

Diagonal segments are produced by ties

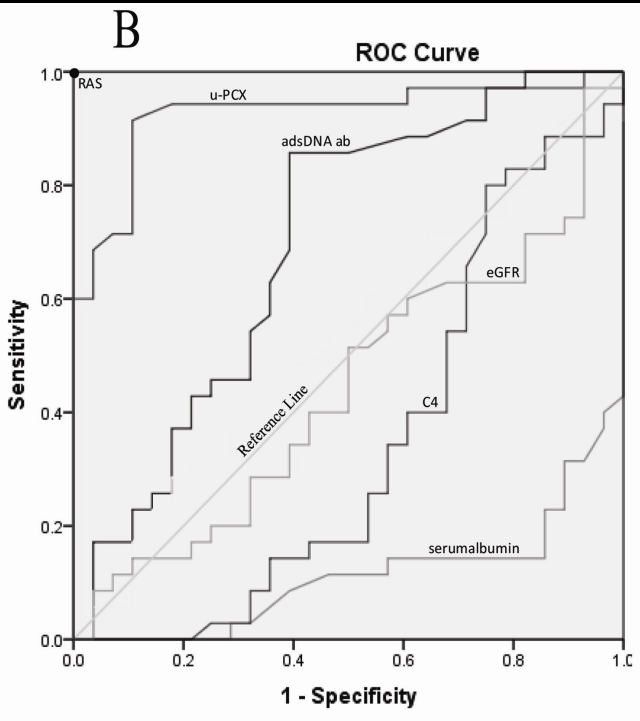

Diagonal segments are produced by ties.

Figure 3. Receiver operating characteristic curves (ROC) of u-PCX and other standard parameters for LN activity.

\section{DISCUSSION}

Many studies have demonstrated the role of PCX in the pathogenesis and progression of glomerular and tubulointerstitial injury in both murine and human LN. According to Vogelmann et al. ${ }^{18}$, cells that stained positive for PCX, stained positive in $30-40 \%$ for other podocyte specific markers as well, while on the other hand cells positive for different podocyte-specific markers stained always positive for PCX. It is important to note that PCX expression is not podocyte specific. 
PCX is also present on endothelial cells, platelets, megakaryocytes, hemangioblasts, and parietal epithelial cells. While it has been shown that PCX-positive cells in the urine do not stain positive for endothelial and mesangial markers ${ }^{19,20}$. In agreement with these data; we reported in our study that u-PCX levels were significantly higher in patient with active LN \& positively correlated with PCR, hematuria, SLEDAI-2K renal \& global scores, ads DNA ab negatively correlated with serum albumin and with no correlation with, leucocyturia, C3 and C4. Furthermore, u-PCX was positively correlated with renal biopsy activity score.

Online with our results; Nakamura et al. ${ }^{8}$ reported that podocyturia diagnosed with the antipodocalyxin antibody could serve as an indicator of active LN and many studies-partially matched with our results- have reported that raised anti-ds DNA abs levels was associated with active renal disease ${ }^{21}$ and that decline in $\mathrm{C} 3$ or $\mathrm{C} 4$ levels coincided with an increase in LN activity ${ }^{22}$. While other studied have reported that, anti-ds DNA abs and serum complement levels were not associated with $\mathrm{LN}$ activity ${ }^{23}$.

Podocyturia is not an indicator for proteinuria ${ }^{20}$, Solórzano et al. ${ }^{24}$ observed that the Podocyturia can be used to monitor the degree of renal involvement in $\mathrm{LN}$, as found in our study. Unlike proteinuria present in active disease and in the chronic stages of glomerular injury, podocyturia seems to be present only in cases of active disease ${ }^{20}$.

Proteinuria in $\mathrm{LN}$ has been associated with immune complex deposition in the subepithelial and subendothelial glomerular capillary wall as well as endocapillary proliferation and inflammation ${ }^{25,26}$. More recently, it has been reported that nephrotic syndrome in patients with LN can occur without immune deposit deposition or inflammation as there is a poor correlation between immune complex deposits and the degree of proteinuria

Histological review of biopsy specimens from these patients show extensive podocyte foot process effacement that is not seen to the same extent in their non-nephrotic counterparts highlighting the role of the podocyte ${ }^{2}$.

It therefore appears that the nephrotic syndrome occurring in $\mathrm{LN}$ involves pathways independent of immune complexes and instead relates to podocyte injury. Anti-ds DNA abs may directly cross-react with podocyte proteins like $\alpha$-actinin- 4 to cause injury ${ }^{27}$.

The effacement of the foot processes as result from podocyte injury has been associated with the development of proteinuria and the nephrotic syndrome. In addition, podocytes have been identified in the population of cells comprising crescentic forms of $\mathrm{LN}^{28}$.
Moreover, a new pathological entity called lupus podocitopathy was introduced by the ISN/RPS in 2003 to describe the recognized phenomenon of apparent minimal change disease in the context of SLE ${ }^{2}$.

In this current study, u-PCX had a good diagnostic profile for early detection of LN activity and better than other parameters of activity, However, it was not as good as SLEDAI-2K renal score and proteinuria for detection of $\mathrm{LN}$ activity. We reported that proteinuria had a significant negative correlation with serum albumin and SLEDAI-2K renal score only in active LN patients which was matched with study reported that Low serum albumin was negatively correlated with LN disease activity ${ }^{29}$.

.Hypoalbuminemia alone will not be the ideal biomarker to identify disease activity in SLE. It may, however, be a useful screening test and guide in patients with LN that would prompt the ordering of a 24-h urine collection for proteinuria ${ }^{29}$. Several factors may affect serum albumin levels like advanced age, proteinuria itself and malnutrition. However, in our study, all patients had a BMI > 18 and therefore a low serum albumin level due to malnutrition was unlikely.

Hypoalbuminemia is common in patients with SLE $^{30.31}$ and it has been correlated to poorer prognoses in SLE and other diseases ${ }^{32}$. Multiple logistic regression analysis showed that only u-PCR and Proteinuria were independent predictors of $\mathrm{LN}$ activity. The use of PCX as a marker for urinary podocytes is further complicated by the fact that the amount of PCX that is shed in the urine cannot originate solely from podocytes ${ }^{33}$. This discrepancy could be explained by PCX shedding from stressed or apoptotic podocytes ${ }^{19}$ and other PCX-positives cells, i.e. parietal epithelial cells ${ }^{34}$. Even so, assessing the total amount of PCX in the urine could be useful as a marker of LN disease activity.

\section{Conclusion}

Our conclusion was that Podocyturia with antipodocalyxin could be served as a marker of LN disease activity.

\section{Acknowledgment}

The authors would like to thank all medical staff in internal medicine department at Riyadh national hospital in Riyadh, KSA for their great support to finish this work

\section{Conflict of Interest}

The authors declare they have no potential conflicts of interest related to the contents of this article. 


\section{REFERENGES}

1. Cervera R, Abarca-Costalago M, Abramovicz D, et al.: Systemic lupus erythematosus in Europe at the change of the millennium: lessons from the "EuroLupus project'”. Autoimmune Rev; 2006, 5:180 186.

2. Kraft SW, Schwartz MM, Korbet SM et al.: Glomerular podocytopathy in patients with systemic lupus erythematosus. J Am Soc Nephrol; 2005, 16: 175-179.

3. Hewitt SM, Dear J, Star RA: Discovery of protein biomarkers for renal diseases. J Am Soc Nephrol; 2004, 15: 1677-1689.

4. Brunner HI, Mueller M, Rutherford C, et al.: Urinary neutrophil gelatinase-associated lipocalin as a biomarker of nephritis in childhood-onset systemic lupus erythematosus. Arthritis Rheum; 2006, 54: 2577- 2584.

5. Kreidberg JA, Donovan MJ, Goldstein SL et al.: Alpha 3 beta 1 integrin has a crucial role in kidney and lung organogenesis. Development; 1996, 122: 3537-3547.

6. Wei C, Moller CC, Altintas MM et al.: Modification of kidney barrier function by the urokinase receptor. Nat Med;2008, 14: 55-63.

7. Mathieson PW. What has the immune system got against the glomerular podocyte? Clin Exp Immunology; 2003, 134:1-5.

8. Nakamura T, Ushiyama C, Suzuki S, et al.: Urinary podocytes for the assessment of disease activity in lupus nephritis. Am J Med Sci; 2000, 320:112-6.

9. Nakamura $\mathrm{T}$, Ushiyama $\mathrm{C}$, Shimada $\mathrm{N}$, et al.: Effect of Cyclophosphamide or azathioprine on urinary podocytes in patients with diffuse proliferative lupus nephritis. Nephron; 2001, 87:192-3.

10. Kerjaschki D, Sharkey DJ, and Farquhar MG.: Identification and characterization of podocalyxinthe major sialoprotein of the renal glomerular Epithelial cell. J Cell Biol; 1984, 98: 1591-1596.

11. Hochberg MC: Updating the American College of Rheumatology revised criteria for the classification of systemic lupus erythematosus. Arthritis Rheum;1997,40: 1725.

12. Bombardier C, Gladman DD, Urowitz MB et al: Derivation of the SLEDAI. A disease activity index for lupus patients. The Committee on Prognosis Studies in SLE. Arthritis Rheum; 1992, 35: 630640.

13. Petri M, Kasitanon N, Lee SS, et al.: Systemic Lupus International Collaborating Clinics Renal Response Exercise (Development of a Renal Activity Score and Renal Response Index). Arthritis Rheum; 2008, 58(6):1784-8.

14. Gladman DD, Ibanez D, Hurwitz MB: Systemic lupus erythematosus disease activity index 2000. J Rheumatol; 2002, 29: 288-291.
15. Weening, J., D’Agati, V., Schwartz, M., et al.: The classification of Glomerulonephritis in systemic lupus erythematosus revisited. J. Am. Soc. Nephrol.; 2004, 15:241-50.

16. Austin H., Muenz L., Joyce, K., et al.: Prognostic factors in lupus nephritis: contribution of renal histologic data. Am. J. Med.; 1983, 75:382-91.

17. Youden WJ: Index for rating diagnostic tests. Cancer; 1950,3: 32-35.

18. Vogelmann SU, Nelson WJ, Myers BD, Lemley $\mathrm{KV}$ : Urinary excretion of viable podocytes in health and renal disease. Am J Physiology Renal Physiology; 2003, 285: F40-F48.

19. Petermann AT, Krofft R, Blonski M, et al.: Podocytes that detach in experimental membranous nephropathy are viable. Kidney Int.; 2003, 64: 1222-1231.

20. Yu D, Petermann A, Kunter U, et al.: Urinary podocyte loss is a more specific marker of ongoing glomerular damage than proteinuria. J Am Soc Nephrol; 2005, 16: 1733-1741.

21- Spronk PE, Bootsma H, Kallenberg CG: Anti-DNA antibodies as early predictor for disease exacerbations in SLE. Guideline for treatment? Clin Rev Allergy Immunology; 1998, 16: 211-218.

22. Ho A, Barr SG, Magder LS: A decrease in complement is associated with increased renal and hematologic activity in patients with systemic lupus erythematosus. Arthritis Rheum; 2001, 44: 23502367.

23. Moroni G, Trendelenburg M, Del Papa N, et al.: Anti-C1q antibodies may help in diagnosing a renal flare in lupus nephritis. Am J Kidney Dis; 2001, 37: 490-498.

24. Solorzano GT, Silva MV, Moreira SR, et al: Urinary protein/creatinine ratio versus 24-hour proteinuria in the evaluation of lupus nephritis. J Bras Nefrol; 2012, 34:64-67.

25. Deji N, Sugimoto T, Kanasaki $M$ et al.: Emerging minimal-change nephrotic syndrome in a patient with chronic mesangial proliferative lupus nephritis. Intern Med; 2007, 46: 991-995

26. Stankeviciute N, JaoW, Bakir A et al.: Mesangial lupus nephritis with associated nephrotic syndrome. J Am Soc Nephrol; 1997, 8: 1199-1204

27. Mason LJ, Ravirajan CT, Rahman A et al.: Is alpha-actinin a target for pathogenic anti-DNA antibodies in lupus nephritis? Arthritis Rheum; 2004, 50: 866-870

28. Sapna T, Martin Z and Jochen R: Role of podocytes in lupus nephritis, Nephrol Dial Transplant; 2009, 24: 3607-3612.

29. YIP. J et al (2010): Serum Albumin as a Marker for Disease Activity in Patients with Systemic Lupus Erythematosus. Journal of Rheumatology; 2010, $37: 8 ; 1667-1672$.

30. Fiehn C, Hajjar Y, Mueller K, et al.: Improved clinical outcome of lupus nephritis during the past decade: Importance of early diagnosis and treatment. Ann Rheum Dis; 2003, 62: 435-439. 
31. Houssiau FA, Vasconcelos C, D'Cruz D, et al.: Early response to immunosuppressive therapy predicts good renal outcome in lupus nephritis.Arthritis Rheum; 2004, 50: 3934-3940.

32. Schwartz N, Su L, Burkly LC, et al. : Urinary TWEAK and the activity of lupus nephritis. J Autoimmun. ; 2006, 27: 242-250.
33. Hara M, Yanagihara T, Kihara I, et al : Apical cell membranes are shed into urine from injured podocytes: a novel phenomenon of podocyte injury. J Am Soc Nephrol; 2005, 16: 408-416.

34. Achenbach J, Mengel M, Tossidou I, et al: Parietal epithelia cells in the urine as a marker of disease activity in glomerular diseases. Nephrol Dial Transplant; 2008, 23: 3138-3145. 